M. Waldschmidt

Nagoya Math. J.

Vol. 155 (1999), 27-53

\title{
DENSITY MEASURE OF RATIONAL POINTS ON ABELIAN VARIETIES
}

\author{
MICHEL WALDSCHMIDT ${ }^{1}$
}

\begin{abstract}
Let $\mathcal{A}$ be a simple Abelian variety of dimension $g$ over $\mathbb{Q}$, and let $\ell$ be the rank of the Mordell-Weil group $\mathcal{A}(\mathbb{Q})$. Assume $\ell \geq 1$. A conjecture of Mazur asserts that the closure of $\mathcal{A}(\mathbb{Q})$ into $\mathcal{A}(\mathbb{R})$ for the real topology contains the neutral component $\mathcal{A}(\mathbb{R})^{0}$ of the origin. This is known only under the extra hypothesis $\ell \geq g^{2}-g+1$. We investigate here a quantitative refinement of this question: for each given positive $h$, the set of points in $\mathcal{A}(\mathbb{Q})$ of Néron-Tate height $\leq h$ is finite, and we study how these points are distributed into the connected component $\mathcal{A}(\mathbb{R})^{0}$. More generally we consider an Abelian variety $\mathcal{A}$ over a number field $K$ embedded in $\mathbb{R}$, and a subgroup $\Gamma$ of $\mathcal{A}(K)$ of sufficiently large rank. The effective result of density we obtain relies on an estimate of Diophantine approximation, namely a lower bound for linear combinations of determinants involving Abelian logarithms.
\end{abstract}

\section{$\S 1$. Introduction}

Let $K$ be a number field with a given real embedding. Let $V$ be a smooth variety over $K$. Denote by $Z$ the closure, for the real topology, of $V(K)$ in $V(\mathbb{R})$. In his paper [9] on the topology of rational points, Mazur assumes that $K=\mathbb{Q}$ and that $V(\mathbb{Q})$ is Zariski dense; he asks whether $Z$ is a union of connected components of $V(\mathbb{R})$. However an example is given in [2] of a smooth surface $V$ over $\mathbb{Q}$, whose $\mathbb{Q}$-rational points are Zariski-dense, but such that the closure $Z$ in $V(\mathbb{R})$ of the set of $\mathbb{Q}$-points is not a union of connected components.

We consider here a quantitative refinement. Assume that $V$ is embedded, as a quasi-projective variety, into a projective space $\mathbb{P}_{N}$ over $K$. Denote by $\mathrm{h}$ the absolute logarithmic height (Weil height) on $\mathbb{P}_{N}(K)$ (see [4], [5, Chap. IV, §1], [6, Chap. III], [7, Chap. II], or [12, Chap. II]):

$$
\mathrm{h}\left(\gamma_{0}: \cdots: \gamma_{N}\right)=\sum_{v \in M_{K}} \frac{\left[K_{v}: \mathbb{Q}_{v}\right]}{[K: \mathbb{Q}]} \log \max \left\{\left|\gamma_{0}\right|_{v}, \ldots,\left|\gamma_{N}\right|_{v}\right\},
$$

\footnotetext{
Received December 16, 1996.

${ }^{1}$ Work partially supported by the Japan Society for the Promotion of Science: grant JSPS 96029
} 
where $M_{K}$ denotes the set of places of $K$ normalized so that the product formula reads

$$
\sum_{v \in M_{K}}\left[K_{v}: \mathbb{Q}_{v}\right] \log |\gamma|_{v}=0 \quad \text { for any } \gamma \in K^{\times} .
$$

Further, for $\zeta=\left(\zeta_{0}: \cdots: \zeta_{N}\right)$ and $\xi=\left(\xi_{0}: \cdots: \xi_{N}\right)$ in $\mathbb{P}_{N}(\mathbb{R})$, write

$$
\operatorname{dist}(\zeta, \xi)=\frac{\max _{0 \leq i, j \leq N}\left|\zeta_{i} \xi_{j}-\zeta_{j} \xi_{i}\right|}{\max _{0 \leq i \leq N}\left|\zeta_{i}\right| \cdot \max _{0 \leq j \leq N}\left|\xi_{j}\right|}
$$

For each sufficiently large real number $H$, define $\eta_{V}(H)$ as follows:

$$
\eta_{V}(H)=\inf \left\{\epsilon>0 ; \begin{array}{l}
\text { for any } \zeta \in Z, \text { there exists } \gamma \in V(K) \\
\text { with } \mathrm{h}(\gamma) \leq \log H \text { and } \operatorname{dist}(\zeta, \gamma) \leq \epsilon
\end{array}\right\} .
$$

Since $V(K)$ is dense in $Z$,

$$
\lim _{H \rightarrow \infty} \eta_{V}(H)=0
$$

An upper bound for $\eta_{V}(H)$ can be considered as a measure of the density in $Z$ of the rational points $V(K)$.

A lower bound for $\eta_{V}(H)$ is easily achieved as follows: denote by $\psi_{V}(H)$ the number of points $\gamma$ in $V(K)$ of height $\mathrm{h}(\gamma) \leq \log H$. Then

$$
\liminf _{H \rightarrow \infty} \psi_{V}(H) \eta_{V}(H)^{\operatorname{dim} V}>0
$$

Since the estimate $\psi_{V}(H) \leq C H^{\kappa}$ always holds, with a constant $C$ which depends only on $V$ and $K$, but not on $H$, and with $\kappa=(\operatorname{dim} V+1)[K: \mathbb{Q}]$, we deduce

$$
\liminf _{H \rightarrow \infty} \eta_{V}(H) \cdot H^{\kappa^{\prime}}>0 \quad \text { with } \kappa^{\prime}=\frac{\kappa}{\operatorname{dim} V} .
$$

In certain cases a sharper lower bound for $\eta_{V}(H)$ is available. For instance when $V$ is an Abelian variety $\mathcal{A}$, if $\ell$ is the rank over $\mathbb{Z}$ of the Mordell-Weil group $\mathcal{A}(K)$, then

$$
\liminf _{H \rightarrow \infty} \eta_{\mathcal{A}}(H) \cdot(\log H)^{\ell / 2 g}>0
$$

Indeed there exists a positive constant $C$ such that, for all $H \geq e$,

$$
\psi_{\mathcal{A}}(H) \leq C(\log H)^{\ell / 2} .
$$


This follows easily from the properties of the Néron-Tate height $\hat{\mathrm{h}}$ associated with the given embedding of $\mathcal{A}$ into $\mathbb{P}_{N}$, which is defined for $\gamma \in \mathcal{A}(K)$ by

$$
\hat{\mathrm{h}}(\gamma)=\lim _{n \rightarrow \infty} \frac{1}{n^{2}} \mathrm{~h}(n \gamma) .
$$

For elliptic curve, see [5, Chap. IV], or [13, Chap. VIII, $\S 6$ and $\S 9]$. For Abelian varieties, see [6, Chap. V], [12, §3.3], [7, Chap. III, §1], or [4, §3].

Since $|\mathrm{h}-\hat{\mathrm{h}}|$ is bounded on $\mathcal{A}(K)$, it does not make a difference for us to replace $\mathrm{h}$ by $\hat{\mathrm{h}}$. Accordingly we define

$$
\hat{\eta}_{\mathcal{A}}(h)=\inf \left\{\epsilon>0 ; \begin{array}{l}
\text { for any } \zeta \in \mathcal{A}(\mathbb{R})^{0}, \text { there exists } \gamma \in \mathcal{A}(K) \\
\text { with } \hat{\mathrm{h}}(\gamma) \leq h \text { and } \operatorname{dist}(\zeta, \gamma) \leq \epsilon
\end{array}\right\}
$$

where $\mathcal{A}(\mathbb{R})^{0}$ denotes the connected component of the origin in $\mathcal{A}(\mathbb{R})$.

Conjecture 1.1. Let $\mathcal{A}$ be a simple Abelian variety of dimension $g$ over a number field $K$ embedded in $\mathbb{R}$. Denote by $\ell$ the rank over $\mathbb{Z}$ of the Mordell-Weil group $\mathcal{A}(K)$. For any $\epsilon>0$, there exists $h_{0}>0$ (which depends only on the Abelian variety $\mathcal{A}$, the real number field $K$ and $\epsilon)$ such that, for any $h \geq h_{0}$,

$$
\hat{\eta}_{\mathcal{A}}(h) \leq h^{-(\ell / 2 g)+\epsilon} .
$$

This means that for sufficiently large $h$, any point in $\mathcal{A}(\mathbb{R})^{0}$ should be at distance $\leq h^{-(\ell / 2 g)+\epsilon}$ of a point of $\mathcal{A}(K)$ of Néron-Tate height $\leq h$. We shall see that this Conjecture 1.1 can be stated in an equivalent way as an estimate of Diophantine approximation, for which we shall produce partial results.

We start with the special case of an elliptic curve. Here is a measure of density for the set of rational points.

ThEOREM 1.2. Let $\mathcal{E}$ be an elliptic curve over a real number field $K$. There exist two positive constants $C_{1}$ and $C_{2}$, which depend only on $\mathcal{E}$ and $K$, such that for any $h \geq e^{e}$,

$$
\hat{\eta}_{\mathcal{E}}(h) \leq C_{1} \exp \left\{-C_{2}(\log h)(\log \log h)^{-1-(2 / \ell)}\right\} .
$$

According to Conjecture 1.1, it should be possible to replace the factor

$$
C_{2}(\log \log h)^{-1-(2 / \ell)} \quad \text { by } \quad(\ell / 2)-\epsilon \quad \text { for } h>h_{0}(\epsilon) .
$$


The proof of Theorem 1.2 (see $\S 3$ ) rests on an estimate, due to $\mathrm{N}$. Hirata-Kohno, on the simultaneous approximation of quotients of elliptic logarithms (namely Theorem 3.2).

As we shall see, the case of an elliptic curve is the only one where the problem is reduced to a lower bound for a linear form in logarithms.

Let $\mathcal{A}$ be a simple Abelian variety of dimension $g$ over a number field $K \subset \mathbb{R}$, with Mordell-Weil group $\mathcal{A}(K)$ of rank $\ell$. If $\ell \geq g^{2}-g+1$, then the closure of $\mathcal{A}(K)$ in $\mathcal{A}(\mathbb{R})$ contains $\mathcal{A}(\mathbb{R})^{0}$. Moreover, if $\ell \geq g^{2}$, then there exists $\gamma \in \mathcal{A}(K) \cap \mathcal{A}(\mathbb{R})^{0}$ such that $\mathbb{Z} \gamma$ is dense in $\mathcal{A}(\mathbb{R})^{0}$ (see Corollary 4.6). Our aim is to provide quantitative refinements to these density results.

We assume that $\mathcal{A}$ is embedded into a projective space $\mathbb{P}_{N}$ over $K$.

ThEOREM 1.3. Assume $\ell \geq 2 g^{2}$. Then there exist two positive constants $C_{1}$ and $C_{2}$ such that, for any $h \geq e$,

$$
\hat{\eta}_{\mathcal{A}}(h) \leq C_{1} \exp \left\{-C_{2}(\log h)^{\theta}\right\} \quad \text { where } \quad \theta=1-\frac{2 g^{2}}{\ell+1} .
$$

The constants $C_{1}$ and $C_{2}$ depend on $\mathcal{A}$ and $K$, as well as on the embedding of $\mathcal{A}$ into $\mathbb{P}_{N}$.

Here is the plan of this paper. The purpose of section 2 is twofold: firstly we state a consequence of Kronecker's Theorem which reduces the density problem to a question of irrationality; secondly we give a transference lemma which reduces the question of density measure to a problem of Diophantine approximation on Abelian varieties. Next in $\S 3$ we discuss the case of elliptic curves. Section 4 is devoted to the qualitative problem on Abelian varieties, and sections 5 and 6 to the quantitative one. The main results of Diophantine approximation (namely Theorems 5.2 and 6.1) are deduced from [19].

Acknowledgements. It is a pleasure for the author to thank Noriko Hirata Kohno for her helpful comments on this paper.

\section{$\S 2$. Kronecker and Khinchine}

Let $E$ be a vector space of dimension $n \geq 1$ over the field $\mathbb{R}$ of real numbers and let $Y$ be a finitely generated subgroup of $E$. From a result of Kronecker one deduces (see [18, Chap. II, Prop. 4.3]): 
LEMMA 2.1. The following conditions are equivalent.

(i) $Y$ is dense in $E$.

(ii) For any vector subspace $V$ of $E$ distinct from $E$, we have

$$
\operatorname{rank}_{\mathbb{Z}}(Y / Y \cap V)>\operatorname{dim}_{\mathbb{R}}(E / V) .
$$

(iii) For any hyperplane $H$ of $E$, we have $\operatorname{rank}_{\mathbb{Z}}(Y / Y \cap H) \geq 2$.

(iv) For any nonzero linear form $\varphi \in \operatorname{Hom}_{\mathbb{R}}(E, \mathbb{R})$, we have $\varphi(Y) \not \subset \mathbb{Z}$.

(v) For any non-trivial character $\chi$ of $E$, we have $\chi(Y) \neq\{1\}$.

(vi) Let $y_{1}, \ldots, y_{\ell}$ be a set of elements of $Y$ which span a subgroup of finite index in $Y$; let $\left(e_{1}, \ldots, e_{n}\right)$ be a basis of $E$ over $\mathbb{R}$; consider the coordinates $u_{i j}$ of $y_{j}$ in this basis:

$$
y_{j}=u_{1 j} e_{1}+\cdots+u_{n j} e_{n}, \quad(1 \leq j \leq \ell)
$$

then for any nonzero $s=\left(s_{1}, \ldots, s_{\ell}\right)$ in $\mathbb{Z}^{\ell}$, the matrix

$$
\mathcal{M}_{s}=\left(\begin{array}{ccc}
u_{11} & \cdots & u_{1 \ell} \\
\vdots & \ddots & \vdots \\
u_{n 1} & \cdots & u_{n \ell} \\
s_{1} & \cdots & s_{\ell}
\end{array}\right)
$$

has $\operatorname{rank} n+1$.

Let $\Omega$ be a lattice in $E$ (which means a discrete $\mathbb{Z}$-subgroup of maximal rank $n)$. Denote by $E^{*}=\operatorname{Hom}_{\mathbb{R}}(E, \mathbb{R})$ the dual vector space of $E$, and by $\Omega^{*}$ the dual lattice of $\Omega$ :

$$
\Omega^{*}=\left\{\varphi \in E^{*} ; \varphi(\Omega) \subset \mathbb{Z}\right\} .
$$

From the equivalence (i) $\Leftrightarrow$ (iv) in Lemma 2.1, it follows that a finitely generated subgroup $Y$ of $E$ which contains $\Omega$ is dense in $E$ if and only if, for any nonzero $\varphi \in \Omega^{*}$, we have $\varphi(Y) \not \subset \mathbb{Z}$. We now give a quantitative version of this statement. For $x \in \mathbb{R}$ we denote by $\|x\|$ the distance to the nearest integer:

$$
\|x\|=\min _{n \in \mathbb{Z}}|x-n|
$$

LEMMA 2.2. Let $\omega_{1}, \ldots, \omega_{n}$ be a basis of the lattice $\Omega$ and let $y_{1}, \ldots, y_{m}$ be elements in E. Let $F$ and $G$ be two monotonically increasing and unbounded real valued functions of a real variable which are inverse of each other:

$$
G \circ F(S)=S \quad \text { for all sufficiently large } S
$$


and

$$
F \circ G(T)=T \text { for all sufficiently large } T \text {. }
$$

Then the two following conditions are equivalent.

(i) There exist three positive constants $c_{1}, c_{2}$ and $S_{0}$ such that, for any nonzero $\varphi \in \Omega^{*}$, if we set

$$
S=\max \left\{\left|\varphi\left(\omega_{1}\right)\right|, \ldots,\left|\varphi\left(\omega_{n}\right)\right| ; S_{0}\right\}
$$

then we have

$$
\max _{1 \leq i \leq m}\left\|\varphi\left(y_{i}\right)\right\| \geq c_{1} / F\left(c_{2} S\right) .
$$

(ii) There exist three positive constants $c_{1}^{\prime}, c_{2}^{\prime}$ and $T_{0}$ such that, for any $x \in E$ and any integer $T \geq T_{0}$, there exist $\omega \in \Omega$ and $\left(t_{1}, \ldots, t_{m}\right) \in \mathbb{Z}^{m}$ which satisfy

$$
\max \left\{\left|t_{1}\right|, \ldots,\left|t_{m}\right|\right\} \leq T \text { and }\left|x-\omega-t_{1} y_{1}-\cdots-t_{m} y_{m}\right| \leq c_{1}^{\prime} / G\left(c_{2}^{\prime} T\right)
$$

Proof. The proof is given in [20, Lemme 3.1], and relies on Khinchine transference Theorem (see also [18, Chap. V, Lemme 4.1]). More precisely, one gets the following explicit estimates.

- If (i) holds, then (ii) is true with

$$
c_{1}^{\prime}=\eta c_{2}|\omega|_{+}, \quad c_{2}^{\prime}=c_{1} / \eta \quad \text { and } \quad T_{0}=\left(1 / c_{2}^{\prime}\right) F\left(c_{2} S_{0}\right)
$$

where

$$
|\omega|_{+}=\left|\omega_{1}\right|+\cdots+\left|\omega_{n}\right| \text { and } \eta=2^{-n-m}((n+m) !)^{2} .
$$

- In the other direction, if (ii) holds, then (i) is true with

$$
c_{1}=c_{2}^{\prime} / 2(n+m), \quad c_{2}=2(n+m) c_{1}^{\prime}|\omega|_{-} \quad \text { and } \quad S_{0}=\left(1 / c_{2}\right) G\left(c_{2}^{\prime} T_{0}\right),
$$

where

$$
|\omega|_{-}=\sup \left\{\left|\omega_{1} z_{1}+\cdots+\omega_{n} z_{n}\right|^{-1} ;\left|z_{1}\right|=\cdots=\left|z_{n}\right|=1\right\}
$$

Remark. Lemma 2.2 deals with the equivalence (i) $\Leftrightarrow$ (iv) of Lemma 2.1. One could also state the result in terms of (vi): the problem is then to produce lower bounds for at least one of the $(n+1) \times(n+1)$ minors of $\mathcal{M}_{s}$ in terms of $\max \left|s_{j}\right|$. 


\section{$\S 3$. Diophantine approximation on elliptic curves}

In this section we deal only with elliptic curves.

Let $\mathcal{E} \subset \mathbb{P}_{2}$ be an elliptic curve over a subfield $K$ of $\mathbb{C}$ given with a Weierstraß model:

$$
\mathcal{E}(K)=\left\{(x: y: t) \in \mathbb{P}_{2}(K) ; y^{2} t=4 x^{3}-g_{2} x t^{2}-g_{3} t^{3}\right\}
$$

where $g_{2}$ and $g_{3}$ are elements of $K$ satisfying $g_{2}^{3} \neq 27 g_{3}^{2}$.

To begin with, assume $K \subset \mathbb{R}$. Then the exponential map of the Lie group $\mathcal{E}(\mathbb{R})$ is the restriction to $\mathbb{R}$ of a Weierstraß $\wp$-function:

$$
\begin{array}{rlc}
\exp _{\mathcal{E}, \mathbb{R}}: \mathbb{R} & \longrightarrow & \mathcal{E}(\mathbb{R}) \\
z & \longmapsto\left(\wp(z): \wp^{\prime}(z): 1\right)
\end{array}
$$

The image $\mathcal{E}(\mathbb{R})^{0}$ of this map is the connected component of the origin in $\mathcal{E}(\mathbb{R})$, while its kernel is of the form $\mathbb{Z} \omega$ for some nonzero period $\omega \in \mathbb{R}$. The induced isomorphism of topological groups between $\mathcal{E}(\mathbb{R})^{0}$ and $\mathbb{R} / \mathbb{Z}$ shows that any finitely generated subgroup of $\mathcal{E}(\mathbb{R})^{0}$ of positive rank is dense. In this case no arithmetic assumption is needed.

We now consider the quantitative density problem. Let $\Gamma$ be a finitely generated subgroup of $\mathcal{E}(\mathbb{R})^{0}$ of rank $\ell$. Let $\gamma_{1}, \ldots, \gamma_{\ell}$ be $\mathbb{Z}$-linearly independent elements in $\Gamma$. For $1 \leq j \leq \ell$, let $y_{j} \in \mathbb{R}$ be an elliptic logarithm of $\gamma_{j}$, viz. a real number such that $\exp _{\mathcal{E}, \mathbb{R}}\left(y_{j}\right)=\gamma_{j}$. From Lemma 2.2, we shall deduce:

LEMMA 3.1. The following properties are equivalent.

(i) For any $\epsilon>0$, there exists a constant $T_{0}>0$, with the following property. For any integer $T \geq T_{0}$ and any $\zeta \in \mathcal{E}(\mathbb{R})^{0}$, there exist rational integers $t_{1}, \ldots, t_{\ell}$ satisfying

$$
\max _{1 \leq j \leq \ell}\left|t_{j}\right| \leq T \quad \text { and } \quad \operatorname{dist}\left(\zeta, t_{1} \gamma_{1}+\cdots+t_{\ell} \gamma_{\ell}\right) \leq T^{-\ell+\epsilon}
$$

(ii) For any $\epsilon>0$ there exists a constant $T_{0}>0$, with the following property. For any integer $T \geq T_{0}$ and any $\xi \in \mathbb{R}$, there exist rational integers $t_{0}, t_{1}, \ldots, t_{\ell}$ satisfying

$$
\max _{1 \leq j \leq \ell}\left|t_{j}\right| \leq T \quad \text { and } \quad\left|\xi-t_{0} \omega-t_{1} y_{1}-\cdots-t_{\ell} y_{\ell}\right| \leq T^{-\ell+\epsilon}
$$


(iii) For any $\epsilon>0$ there exists a constant $Q>0$, with the following property: for any rational integers $q, p_{1}, \ldots, p_{\ell}$ with $q \geq Q$,

$$
\max _{1 \leq j \leq \ell}\left|\frac{y_{j}}{\omega}-\frac{p_{j}}{q}\right|>q^{-(1 / \ell)-1-\epsilon} .
$$

Moreover, if the field $K$ is an algebraic extension of $\mathbb{Q}$ and if $\Gamma$ is contained in $\mathcal{E}(K)$, then these properties are also equivalent to the next one:

(iv) For any $\epsilon>0$ there exists a constant $h_{0}>0$ such that, for any $h \geq h_{0}$ and any $\zeta \in \mathcal{E}(\mathbb{R})^{0}$, there exists $\gamma \in \Gamma$ with $\hat{\mathrm{h}}(\gamma) \leq h$ and

$$
\operatorname{dist}(\zeta, \gamma) \leq h^{-(\ell / 2)+\epsilon}
$$

In each of these properties, the "constants" are supposed to depend only on $\epsilon, g_{2}, g_{3}, K$ and $y_{1}, \ldots, y_{\ell}$ - notice that the real number $\omega$ depends only on $g_{2}$ and $g_{3}$.

Proof. There is no loss (apart from the constants, which we do not compute explicitly here) to replace if necessary $\Gamma$ by a subgroup of finite index. Hence one may assume $\Gamma=\mathbb{Z} \gamma_{1}+\cdots+\mathbb{Z} \gamma_{\ell}$.

For the proof of $(\mathrm{i}) \Leftrightarrow\left(\right.$ ii), write any $\zeta \in \mathcal{E}(\mathbb{R})^{0}$ as $\exp _{\mathcal{E}, \mathbb{R}}(\xi)$ for some $\xi \in \mathbb{R}$. Also write any $\gamma \in \Gamma$ as $\exp _{\mathcal{E}, \mathbb{R}}\left(t_{1} y_{1}+\cdots+t_{\ell} y_{\ell}\right)$ for some $\left(t_{1}, \ldots, t_{\ell}\right) \in$ $\mathbb{Z}^{\ell}$. Up to a (multiplicative) constant, $\operatorname{dist}(\zeta, \gamma)$ is nothing else than

$$
\min _{t_{0} \in \mathbb{Z}}\left|\xi-t_{0} \omega-t_{1} y_{1}-\cdots-t_{\ell} y_{\ell}\right| \text {. }
$$

For the proof of the equivalence between (ii) and (iii), use Lemma 2.2 with

$$
E=\mathbb{R}, \quad n=1, \quad \Omega=\mathbb{Z} \omega, \quad F(S)=S^{(1 / \ell)+\epsilon_{1}} \quad \text { and } \quad G(T)=T^{\ell-\epsilon_{2}},
$$

where $\epsilon_{1}$ and $\epsilon_{2}$ are related by $\epsilon_{1} \ell-\epsilon_{2} / \ell=\epsilon_{1} \epsilon_{2}$.

Finally, the correspondence between $h$ in (iv) and $T$ in (i) is $h=T^{2}$.

It seems reasonable to conjecture that the equivalent properties stated in Lemma 3.1 always hold when the field $K$ is a (real) number field. This would imply Conjecture 1.1 for $g=1$ (just take for $\gamma_{1}, \ldots, \gamma_{\ell}$ a basis of the Mordell-Weil group $\mathcal{E}(K)$ ). Moreover, as far as assertion (iii) is concerned, one expects that such an estimate should hold for any number field $K$, not only real ones. In this direction, the best known estimate for the simultaneous approximation of the numbers $y_{j} / \omega$ is due to N. Hirata-Kohno (cf. [3]): 
TheOREM 3.2. (Hirata-Kohno) Let $\mathcal{E}$ be an elliptic curve over a number field $K$. Let $\omega \in \mathbb{C}$ be a non-zero period of $\exp _{\mathcal{E}}$. Let $y_{1}, \ldots$, y be complex numbers such that $\exp _{\mathcal{E}}\left(y_{j}\right) \in \mathcal{E}(K)$ for $1 \leq j \leq \ell$. Assume the numbers $\omega, y_{1}, \ldots, y_{\ell}$ are linearly independent over $\mathbb{Q}$. Then there exists a constant $c$, which depends only on $g_{2}, g_{3}, \omega, K$ and $y_{1}, \ldots, y_{\ell}$, such that, for any $\left(q, p_{1}, \ldots, p_{\ell}\right) \in \mathbb{Z}^{\ell+1}$ with $q>e^{e}$,

$$
\max _{1 \leq j \leq \ell}\left|\frac{y_{j}}{\omega}-\frac{p_{j}}{q}\right| \geq \exp \left\{-c(\log q)(\log \log q)^{1+(2 / \ell)}\right\} .
$$

We now deduce from Theorem 3.2 a stronger statement than Theorem 1.2 (we do not restrict the discussion to the full Mordell-Weil group here).

COROLlary 3.3. Let $\mathcal{E}$ be an elliptic curve over a real number field $K$ and let $\Gamma$ be a subgroup of $\mathcal{E}(K)$ of positive rank $\ell$. There exists a constant $C>0$ such that, for any $h \geq e^{e}$ and any $\zeta \in \mathcal{E}(\mathbb{R})^{0}$, there exists $\gamma \in \Gamma$ with $\hat{\mathrm{h}}(\gamma) \leq h$ and

$$
\operatorname{dist}(\zeta, \gamma) \leq \exp \left\{-C(\log h)(\log \log h)^{-1-(2 / \ell)}\right\}
$$

Proof. We use the implication (i) $\Rightarrow$ (ii) of Lemma 2.2 with

$$
F(S)=\exp \left\{(\log S)(\log \log S)^{1+(2 / \ell)}\right\} \quad \text { for } S \geq e^{e} .
$$

Then the inverse function $G$ is bounded from below by

$$
G(T) \geq \exp \left\{(\log T)(\log \log T)^{-1-(2 / \ell)}\right\} \quad \text { for } T \geq e^{e} .
$$

\section{$\S 4$. Irrationality, transcendence and density}

Let $\mathcal{A}$ be an Abelian variety of dimension $g$ defined over a subfield $K$ of $\mathbb{C}$. We denote by $T_{\mathcal{A}}(\mathbb{C})$ the tangent space at the origin of $\mathcal{A}$, by

$$
\exp _{\mathcal{A}}: T_{\mathcal{A}}(\mathbb{C}) \longrightarrow \mathcal{A}(\mathbb{C})
$$

the exponential map of $\mathcal{A}(\mathbb{C})$, and by $\Omega_{\mathcal{A}}$ its kernel, which is a discrete subgroup of $T_{\mathcal{A}}(\mathbb{C})$ of rank $2 g$ over $\mathbb{Z}$. If $K$ is a subfield of $\mathbb{R}$, then the exponential map of the real Lie group $\mathcal{A}(\mathbb{R})$ is the restriction

$$
\exp _{\mathcal{A}, \mathbb{R}}: T_{\mathcal{A}}(\mathbb{R}) \longrightarrow \mathcal{A}(\mathbb{R})
$$


of $\exp _{\mathcal{A}}$ to $T_{\mathcal{A}}(\mathbb{R})$. Its image is $\mathcal{A}(\mathbb{R})^{0}$ and its kernel

$$
\Omega_{\mathcal{A}, \mathbb{R}}=\Omega_{\mathcal{A}} \cap T_{\mathcal{A}}(\mathbb{R})
$$

is a discrete subgroup of $T_{\mathcal{A}}(\mathbb{R})$ of rank $g$ over $\mathbb{Z}$.

We denote by

$$
\mathcal{L}_{\mathcal{A}}(K)=\exp _{\mathcal{A}}^{-1}(\mathcal{A}(K))
$$

the $\mathbb{Z}$-module of Abelian logarithms of rational points in $\mathcal{A}(K)$. If $K$ is algebraically closed, then the group $\mathcal{A}(K)$ is divisible, hence $\mathcal{L}_{\mathcal{A}}(K)$ is a $\mathbb{Q}$-vector space.

The following result is a consequence of Lemma 2.1.

Lemma 4.1. Let $\mathcal{A}$ be an Abelian variety defined over $\mathbb{R}$. Let $\Gamma$ be a finitely generated subgroup of $\mathcal{A}(\mathbb{R})^{0}$. Then the following properties are equivalent.

(i) The subgroup $\Gamma$ is dense in $\mathcal{A}(\mathbb{R})^{0}$.

(ii) Define $Y=\exp _{\mathcal{A}, \mathbb{R}}^{-1}(\Gamma)$. Then for any nonzero linear form $\varphi \in$ $\operatorname{Hom}_{\mathbb{R}}\left(T_{\mathcal{A}}(\mathbb{R}), \mathbb{R}\right)$, we have $\varphi(Y) \not \subset \mathbb{Q}$.

(iii) Let $\omega_{1}, \ldots, \omega_{g}$ be linearly independent elements of $\Omega_{\mathcal{A}, \mathbb{R}}$. Let $y_{1}, \ldots$, $y_{\ell}$ be elements in $T_{\mathcal{A}}(\mathbb{R})$ whose exponentials $\exp _{\mathcal{A}, \mathbb{R}}\left(y_{j}\right),(1 \leq j \leq \ell)$ span a subgroup of finite index of $\Gamma$. Further, consider the coordinates of $y_{j}$ in the basis $\omega_{1}, \ldots, \omega_{g}$ of $T_{\mathcal{A}}(\mathbb{R})$ :

$$
y_{j}=u_{1 j} \omega_{1}+\cdots+u_{g j} \omega_{g}, \quad(1 \leq j \leq \ell) .
$$

Then for any $\left(\sigma_{1}, \ldots, \sigma_{g}, s_{1}, \ldots, s_{\ell}\right)$ in $\mathbb{Z}^{g+\ell}$ with $\left(\sigma_{1}, \ldots, \sigma_{g}\right) \neq(0, \ldots, 0)$, the matrix

$$
\left(\begin{array}{cccccc} 
& & & u_{11} & \cdots & u_{1 \ell} \\
& I_{g} & & \vdots & \ddots & \vdots \\
& & & u_{g 1} & \cdots & u_{g \ell} \\
\sigma_{1} & \cdots & \sigma_{g} & s_{1} & \cdots & s_{\ell}
\end{array}\right)
$$

has rank $g+1$.

For instance a subgroup $\mathbb{Z} \gamma$ of $\mathcal{A}(\mathbb{R})^{0}$ is dense if and only if $\gamma=$ $\exp _{\mathcal{A}, \mathbb{R}}(y)$ with $y=\vartheta_{1} \omega_{1}+\cdots+\vartheta_{g} \omega_{g}$ and $1, \vartheta_{1}, \ldots, \vartheta_{g}$ linearly independent over $\mathbb{Q}$.

It seems reasonable to hope that these properties are true when $\mathcal{A}$ is simple and $K$ is a (real) number field. This would imply the following 
stronger version of Mazur's conjecture for Abelian varieties: for a simple Abelian variety $\mathcal{A}$ over a real number field $K$, the closure in $\mathcal{A}(\mathbb{R})$ of any subgroup of positive rank of $\mathcal{A}(K)$ contains $\mathcal{A}(\mathbb{R})^{0}$.

Condition (iii) suggests a problem of irrationality: for any finitely generated subgroup $Y$ of $\mathcal{L}_{\mathcal{A}}(K)$ of positive rank and any nonzero linear form $\varphi \in \operatorname{Hom}_{\mathbb{R}}\left(T_{\mathcal{A}}(\mathbb{R}), \mathbb{R}\right)$, prove that one at least of the numbers $\varphi(y),(y \in Y)$ is irrational. It is natural to extend this question to a transcendence problem: prove that one at least of the numbers $\varphi(y),(y \in Y)$ is transcendental. In this later case it is also natural to consider a nonzero linear form $\varphi$ on the complex vector space $T_{\mathcal{A}}(\mathbb{C})$ (the number field $K$ need not be real). More generally we produce a lower bound for the dimension of the $\overline{\mathbb{Q}}$-vector space spanned by $\varphi(Y)$ in $\mathbb{C}$.

THEOREM 4.2. Let $\mathcal{A}$ be a simple Abelian variety over a number field $K, Y$ be a finitely generated subgroup of $\mathcal{L}_{\mathcal{A}}(\overline{\mathbb{Q}})$ of rank $m$ and $\varphi \in$ $\operatorname{Hom}_{\mathbb{C}}\left(T_{\mathcal{A}}(\mathbb{C}), \mathbb{C}\right)$ a nonzero linear form. Define

$$
\kappa=\operatorname{rank}_{\mathbb{Z}}\left(\Omega_{\mathcal{A}} \cap \operatorname{Ker} \varphi\right)
$$

Let $r$ be the dimension of the $\overline{\mathbb{Q}}$-vector space spanned by $\varphi(Y)$. Then

$$
m \leq(2 g-\kappa)(g+r-1)
$$

The number $\kappa$ lies in the range $0 \leq \kappa \leq g-1$. If there exist $k$ periods $\omega_{1}, \ldots, \omega_{k}$ in $\Omega_{\mathcal{A}}$ which are linearly independent and such that $\varphi\left(\omega_{i}\right) \in \mathbb{Q}$ for $1 \leq i \leq k$, then $\kappa \geq k-1$. In other words

$$
\kappa+1 \geq \operatorname{rank}_{\mathbb{Z}}\left(\Omega_{\mathcal{A}} \cap \varphi^{-1}(\mathbb{Q})\right)
$$

From Theorem 4.2 (with $r=0$ ) one deduces:

$$
\text { if } m>(2 g-\kappa)(g-1) \text {, then } \varphi(Y) \neq\{0\}
$$

This means that $\varphi(Y)$ has rank $\geq m-(2 g-\kappa)(g-1)$. Hence from (4.4) we derive

$$
\text { if } m>(2 g-\kappa)(g-1)+1 \text {, then } \varphi(Y) \not \subset \mathbb{Q} \text {. }
$$

This statement includes the following density result (see [17, Cor. 4.1]; see also [18, Chap. IV, §4]): 
COROLlary 4.6. Let $\mathcal{A}$ be a simple Abelian variety over a real number field $K$ and let $\Gamma$ be a subgroup of $\mathcal{A}(K)$ of rank $\ell$. If $\ell \geq g^{2}-g+1$, then the closure of $\Gamma$ in $\mathcal{A}(\mathbb{R})$ contains $\mathcal{A}(\mathbb{R})^{0}$. Further, if $\ell \geq g^{2}$, then there exists a subgroup of $\Gamma$ of rank 1 which is dense in $\mathcal{A}(\mathbb{R})^{0}$.

Proof. Using Lemma 4.1, we deduce the first part from (4.3) and (4.5) with $Y=\exp _{\mathcal{A}, \mathbb{R}}^{-1}(\Gamma), m=\ell+g$ and $\kappa=g-1$. The second part is a consequence of the first one by means of Lemma 3.2 of [10].

We also deduce from Theorem 4.2 (now with $r=1$ ):

$$
\text { If } m>(2 g-\kappa) g \text {, then } \varphi(Y) \not \subset \overline{\mathbb{Q}} \text {. }
$$

In case $g=1, \kappa=1$, the statement (4.7) just means that $u / \omega$ is transcendental if $u \in \mathcal{L}_{\mathcal{E}}(\overline{\mathbb{Q}})$ is a logarithm of a point of infinite order. This is a result of Schneider's [11]. For $g=1$ again, but $\kappa=0$, the condition $m \geq 3$ cannot be improved when $\mathcal{E}$ is a CM elliptic curve: in this case the result (again due to Schneider) states that the quotient of two elliptic logarithms of points of infinite order is either in the field of endomorphisms, or else is transcendental. These are the only cases where (4.7) is know to be optimal. For instance in case $g=1, \kappa=0$ and $\mathcal{E}$ without complex multiplication, Schneider's result shows that the assumption $m \geq 2$ is sufficient to imply $\varphi(Y) \not \subset \overline{\mathbb{Q}}$.

Finally, Theorem 4.2 (in case $g=1$ ) contains the following result due to D. W. Masser [8]: if $\mathcal{E}$ is an elliptic curve defined over the field $\overline{\mathbb{Q}}$ with complex multiplication, and if $y_{1}, \ldots, y_{\ell}$ are in $\mathcal{L}_{\mathcal{E}}(\overline{\mathbb{Q}})$ and linearly independent over the field of endomorphisms of $\mathcal{E}$, then $y_{1}, \ldots$, ye are linearly independent over $\overline{\mathbb{Q}}$.

The similar result (due to Bertrand and Masser [1]) in the non-CM case does not seem to follow from Theorem 4.2, nor do the inhomogeneous linear independence results.

We now prove Theorem 4.2. We shall use the following special case of the Theorem of the algebraic subgroup (see [15] and [16]).

THEOREM 4.8. Let $\mathcal{A}$ be a simple Abelian variety of dimension $g$ over a subfield $K$ of $\overline{\mathbb{Q}}$, let $d_{0}$ be a nonnegative integer, and let $\mathcal{V}$ be a vector subspace of $\mathbb{C}^{d_{0}} \times T_{\mathcal{A}}(\mathbb{C})$. Assume

$$
\mathcal{V} \cap\left(\overline{\mathbb{Q}}^{d_{0}} \times\{0\}\right)=\{0\} \quad \text { and } \quad \mathcal{V} \not \supset\{0\} \times T_{\mathcal{A}}(\mathbb{C})
$$


Define

$$
\kappa=\operatorname{rank}_{\mathbb{Z}}\left(\mathcal{V} \cap\left(\{0\} \times \Omega_{\mathcal{A}}\right)\right)
$$

Then

$$
\operatorname{dim}_{\mathbb{Q}}\left(\mathcal{V} \cap\left(\overline{\mathbb{Q}}^{d_{0}} \times \mathcal{L}_{\mathcal{A}}(\overline{\mathbb{Q}})\right)\right) \leq(2 g-\kappa)\left(d_{0}+g-1\right)
$$

Proof of Theorem 4.2. Let $\left(\xi_{1}, \ldots, \xi_{r}\right)$ be a basis over $\overline{\mathbb{Q}}$ of the vector field spanned by $\varphi(Y)$ in $\mathbb{C}$. For $y \in Y$, denote by $\psi(y) \in \overline{\mathbb{Q}}^{r}$ the vector whose components are the coordinates of $\varphi(y)$ in this basis. This defines a homomorphism of additive groups $\psi: Y \rightarrow \overline{\mathbb{Q}}^{r}$.

Let $\mathcal{V}$ be the hyperplane of $\mathbb{C}^{r} \times T_{\mathcal{A}}(\mathbb{C})$ of equation

$$
\xi_{1} z_{1}+\cdots+\xi_{r} z_{r}=\varphi(z), \quad\left(z_{1}, \ldots, z_{r}, z\right) \in \mathbb{C}^{r} \times T_{\mathcal{A}}(\mathbb{C})
$$

Since $\xi_{1}, \ldots, \xi_{r}$ are linearly independent over $\overline{\mathbb{Q}}$, we have

$$
\mathcal{V} \cap\left(\overline{\mathbb{Q}}^{r} \times\{0\}\right)=\{0\}
$$

Since the form $\varphi$ is not zero, we have

$$
\mathcal{V} \not \supset\{0\} \times T_{\mathcal{A}}(\mathbb{C}) \text {. }
$$

The number $\kappa$ in Theorem 4.2 is the same as in Theorem 4.8 , because

$$
\mathcal{V} \cap\left(\{0\} \times \Omega_{\mathcal{A}}\right)=(\{0\} \times \operatorname{Ker} \varphi) \cap\left(\{0\} \times \Omega_{\mathcal{A}}\right)
$$

Moreover, for each $y \in Y$, we have

$$
(\psi(y), y) \in \mathcal{V} \cap\left(\overline{\mathbb{Q}}^{r} \times \mathcal{L}_{\mathcal{A}}(\overline{\mathbb{Q}})\right) .
$$

Finally, we use Theorem 4.8 with $d_{0}=r$ and conclude

$$
\operatorname{rank}_{\mathbb{Z}}(Y) \leq \operatorname{dim}_{\mathbb{Q}}\left(\mathcal{V} \cap\left(\overline{\mathbb{Q}}^{r} \times \mathcal{L}_{\mathcal{A}}(\overline{\mathbb{Q}})\right)\right) \leq(2 g-\kappa)(g+r-1)
$$

Remark. There is a corresponding multiplicative story which relies on a variant of Theorem 4.8 , where the algebraic group $\mathbb{G}_{\mathrm{a}}^{d_{0}} \times \mathcal{A}$ is replaced by $\mathbb{G}_{\mathrm{m}}^{d_{1}} \times \mathcal{A}$. 


\section{$\S 5$. Rational Diophantine approximation on Abelian varieties}

Let $\mathcal{A}$ an Abelian variety of dimension $g$ over a subfield $K$ of $\mathbb{R}$. Fix an embedding of $\mathcal{A}$ in a projective space over $K$ as well as a norm $|\cdot|$ on the tangent space at the origin $T_{\mathcal{A}}(\mathbb{R})$. Let $\omega_{1}, \ldots, \omega_{g}$ be a basis of $\Omega_{\mathcal{A}, \mathbb{R}}$. Further, let $\gamma_{1}, \ldots, \gamma_{\ell}$ be $\mathbb{Z}$-linearly independent elements in $\mathcal{A}(\mathbb{R})^{0}$. For $1 \leq j \leq \ell$, let $y_{j} \in T_{\mathcal{A}}(\mathbb{R})$ be an Abelian logarithm of $\gamma_{j}$, viz. an element of $T_{\mathcal{A}}(\mathbb{R})$ such that $\exp _{\mathcal{A}, \mathbb{R}}\left(y_{j}\right)=\gamma_{j}$.

The proof of Lemma 3.1 extends to the case $g \geq 1$ and yields the following.

LEMMA 5.1. Let $\theta$ be a positive real number. The following properties are equivalent.

(i) There exists a constant $C>0$, with the following property. For any positive integer $T$ and any $\zeta \in \mathcal{A}(\mathbb{R})^{0}$, there exist rational integers $t_{1}, \ldots, t_{\ell}$ satisfying

$$
\max _{1 \leq j \leq \ell}\left|t_{j}\right| \leq T \quad \text { and } \quad \operatorname{dist}\left(\zeta, t_{1} \gamma_{1}+\cdots+t_{\ell} \gamma_{\ell}\right) \leq C T^{-\theta}
$$

(ii) There exists a constant $C>0$, with the following property. For any positive integer $T$ and any $\xi \in T_{\mathcal{A}}(\mathbb{R})$, there exist rational integers $\tau_{1}, \ldots, \tau_{g}$, $t_{1}, \ldots, t_{\ell}$ satisfying

$$
\max _{1 \leq j \leq \ell}\left|t_{j}\right| \leq T \quad \text { and } \quad\left|\xi-\tau_{1} \omega_{1}-\cdots-\tau_{g} \omega_{g}-t_{1} y_{1}-\cdots-t_{\ell} y_{\ell}\right| \leq C T^{-\theta}
$$

(iii) There exists a constant $C>0$ with the following property. If $\varphi \in$ $\operatorname{Hom}_{\mathbb{R}}\left(T_{\mathcal{A}}(\mathbb{R}), \mathbb{R}\right)$ is a nonzero linear form such that $\varphi\left(\omega_{i}\right) \in \mathbb{Z}$ for $1 \leq i \leq g$, then

$$
\max _{1 \leq j \leq \ell}\left\|\varphi\left(y_{j}\right)\right\| \geq C S^{-1 / \theta} \quad \text { where } S=\max _{1 \leq i \leq g}\left|\varphi\left(\omega_{i}\right)\right| .
$$

Moreover, if the field $K$ is an algebraic extension of $\mathbb{Q}$ and if the group $\Gamma=\mathbb{Z} \gamma_{1}+\cdots+\mathbb{Z} \gamma_{\ell}$ is contained in $\mathcal{A}(K)$, then these properties are also equivalent to the next one:

(iv) There exists a constant $C>0$ such that, for any $h \geq 1$ and any $\zeta \in \mathcal{A}(\mathbb{R})^{0}$, there exists $\gamma \in \Gamma$ with $\hat{\mathrm{h}}(\gamma) \leq h$ and

$$
\operatorname{dist}(\zeta, \gamma) \leq C h^{-\theta / 2}
$$


In each of these properties, the "constants" depend only on $\theta, \mathcal{A}, K$, $\omega_{1}, \ldots, \omega_{g}, y_{1}, \ldots, y_{\ell}$, as well as the norm on $T_{\mathcal{A}}(\mathbb{R})$ for property (iii) and the embedding of $\mathcal{A}$ into $\mathbb{P}_{N}$ for properties (i) and (iv).

The best possible value (i.e., the largest one) of $\theta$ for which the properties of Lemma 5.1 can hold is $\theta=\ell / g$ : this is the "Dirichlet exponent" of $[14$, p. 36].

It seems reasonable to expect that the equivalent properties stated in Lemma 5.1 hold for any $\theta<\ell / g$ when the variety $\mathcal{A}$ is simple and $K$ is a (real) number field. This would clearly imply Conjecture 1.1.

In order to check condition (iii) of Lemma 5.1, one needs to produce lower bounds for numbers of the form

$$
s_{0}+s_{1} \vartheta_{1}+\cdots+s_{\ell} \vartheta_{\ell}
$$

where $\vartheta_{1}, \ldots, \vartheta_{\ell}$ are certain determinants of matrices; the entries of these matrices are coordinates of Abelian logarithms of algebraic points on the variety. In this direction we shall prove two estimates of simultaneous Diophantine approximation. The first one (Corollary 5.3) yields lower bounds for homogeneous linear forms. The second one (Theorem 6.1) is an inhomogeneous estimate of approximation by algebraic numbers.

In the next statements, the Abelian varieties are defined over a number field which is embedded in $\mathbb{C}$. For such an Abelian variety $\mathcal{A}$, we fix a basis $\left(e_{1}, \ldots, e_{g}\right)$ of the tangent space at the origin $T_{\mathcal{A}}(\mathbb{C})$, as well as a norm $|\cdot|$ on the same, namely

$$
|z|=\max \left\{\left|z_{1}\right|, \ldots,\left|z_{g}\right|\right\} \quad \text { for } z=z_{1} e_{1}+\cdots+z_{g} e_{g} \in T_{\mathcal{A}}(\mathbb{C})
$$

Further, for a linear form $\varphi \in \operatorname{Hom}_{\mathbb{C}}\left(T_{\mathcal{A}}(\mathbb{C}), \mathbb{C}\right)$, we define

$$
\mathrm{N}(\varphi)=\max \left\{\left|\varphi\left(e_{1}\right)\right|, \ldots,\left|\varphi\left(e_{g}\right)\right|\right\} .
$$

ThEOREM 5.2. $\quad$ Let $\mathcal{A}$ be a simple Abelian variety over a number field $K_{0}$ of dimension $g$ and let $m$ be a positive integer. For any sufficiently large real number $c_{0}$ the following property holds. Let $K$ be a finite extension of $K_{0}$, with $[K: \mathbb{Q}]=D$. Let $y_{1}, \ldots, y_{m}$ be elements of $\mathcal{L}_{\mathcal{A}}(K)$. For $1 \leq j \leq m$ define $\gamma_{j}=\exp _{\mathcal{A}} y_{j} \in \mathcal{A}(K)$. Define a real number $A \geq e$ by

$$
\log A=\max _{1 \leq j \leq m} \max \left\{1, \mathrm{~h}\left(\gamma_{j}\right),(e / D)\left|y_{j}\right|^{2}\right\}
$$


Let $S$ be a positive integer and $\varphi \in \operatorname{Hom}_{\mathbb{C}}\left(T_{\mathcal{A}}(\mathbb{C}), \mathbb{C}\right)$ a nonzero linear form. Define

$$
V=c_{0}\left(c_{0}^{2} S^{2} D \log A\right)^{g}
$$

and

$$
\mathcal{S}=\left\{s=\left(s_{1}, \ldots, s_{m}\right) \in \mathbb{Z}^{m} ; \begin{array}{l}
\left|s_{j}\right|<S,(1 \leq j \leq m) ; \\
\left|\varphi\left(s_{1} y_{1}+\cdots+s_{m} y_{m}\right)\right| \leq \mathrm{N}(\varphi) e^{-V}
\end{array}\right\} .
$$

Then the number of elements of the set

$$
\Sigma=\left\{s_{1} \gamma_{1}+\cdots+s_{m} \gamma_{m} ; s \in \mathcal{S}\right\} \subset \mathcal{A}(K)
$$

is bounded by

$$
\operatorname{Card} \Sigma \leq c_{0}^{2} V^{g-1}
$$

Proof. The basic tool for the proof of Theorem 5.2 will be the effective version of the algebraic subgroup Theorem given in [19].

We assume that the hypotheses of Theorem 5.2 are satisfied. There is no loss of generality to assume $\mathrm{N}(\varphi)=1$ and $\varphi\left(e_{g}\right)=-1$ :

$$
\varphi\left(z_{1} e_{1}+\cdots+z_{g} e_{g}\right)=\vartheta_{1} z_{1}+\cdots+\vartheta_{g-1} z_{g-1}-z_{g},
$$

with $\max \left\{\left|\vartheta_{1}\right|, \ldots,\left|\vartheta_{g-1}\right|\right\} \leq 1$.

We denote by $c_{0}$ a sufficiently large number, which depends on $m$, on the Abelian variety $\mathcal{A}$, on the chosen basis of $T_{\mathcal{A}}(\mathbb{C})$ and on the number field $K_{0}$, but not on $K, y_{1}, \ldots, y_{m}$, nor on $\vartheta_{1}, \ldots, \vartheta_{g-1}$. In particular $c_{0}$ does not depend on the parameters $D, A$ and $S$.

We use the notation of Theorem 2.1 of [19] with

$$
\begin{gathered}
G=\mathcal{A}, \quad d_{0}=d_{1}=0, \quad d_{2}=d=g, \\
W=\mathcal{W}^{\prime}=\{0\}, \quad \ell_{0}=0, \quad r=r_{3}=g-1, \quad r_{1}=r_{2}=0,
\end{gathered}
$$

while $\mathcal{V}^{\prime}$ is the hyperplane $\operatorname{Ker} \varphi$ of $T_{\mathcal{A}}(\mathbb{C})$. Further we define

$$
U=V / c_{0}, \quad \log A_{1}=c_{0} S^{2} \log A, \quad E=e, \quad M=\operatorname{Card} \mathcal{S}
$$

and

$$
T_{1}=\left[U / D \log A_{1}\right]=\left[U / c_{0} S^{2} D \log A\right]
$$

Since

$$
\left(U / c_{0} S^{2} D \log A\right)^{g}=c_{0} V^{g-1},
$$


one deduces

$$
T_{1}^{g} \leq c_{0} V^{g-1} \leq\left(T_{1}+1\right)^{g} .
$$

For $1 \leq j \leq m$ define $y_{j}^{\prime} \in \operatorname{Ker} \varphi$ by

$$
y_{j}^{\prime}=y_{j}+\varphi\left(y_{j}\right) e_{g}
$$

For each $s \in \mathcal{S}$, define two elements of $T_{\mathcal{A}}(\mathbb{C})$ :

$$
\eta_{s}=s_{1} y_{1}+\cdots+s_{m} y_{m}, \quad \eta_{s}^{\prime}=s_{1} y_{1}^{\prime}+\cdots+s_{m} y_{m}^{\prime},
$$

so that, for any $s \in \mathcal{S}$,

$$
\left|\eta_{s}-\eta_{s}^{\prime}\right|=\left|\varphi\left(s_{1} y_{1}+\cdots+s_{m} y_{m}\right)\right| \leq e^{-V} .
$$

Since the only connected algebraic subgroup of $\mathcal{A}$, distinct from $\mathcal{A}$, is $\{0\}$, one deduces from Theorem 2.1 in [19]:

$$
\operatorname{Card} \Sigma \leq c_{0} T_{1}^{g} \leq c_{0}^{2} V^{g-1}
$$

Theorem 5.2 is the Abelian counterpart to Theorem 4.3 of [20], which deals with $\mathbb{G}_{\mathrm{m}}^{d}$. We now deduce from Theorem 5.2 Abelian analogues of Theorem 1.2 and Corollary 1.3 of [20]. Theorem 6.1 in the next section is an Abelian analogue of Theorem 1.4 of [20].

Corollary 5.3. Let $\mathcal{A}$ be a simple Abelian variety of dimension $g$ over a number field $K_{0}$ and let $\ell$ be a positive integer with $\ell>2 g(g-1)$. There exists a constant $c>0$ with the following property. Let $K$ be a finite

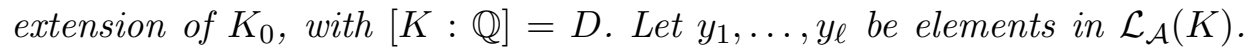
For $1 \leq j \leq \ell$ define $\gamma_{j}=\exp _{\mathcal{A}} y_{j}$ and assume that $\gamma_{1}, \ldots, \gamma_{\ell}$ are $\mathbb{Z}$-linearly independent in $\mathcal{A}(K)$. Define a real number $A \geq$ e by

$$
\log A=\max _{1 \leq j \leq \ell} \max \left\{1, \mathrm{~h}\left(\gamma_{j}\right),(e / D)\left|y_{j}\right|^{2}\right\} .
$$

Let $\varphi \in \operatorname{Hom}_{\mathbb{C}}\left(T_{\mathcal{A}}(\mathbb{C}), \mathbb{C}\right)$ be a nonzero linear form. Then

$$
\max _{1 \leq j \leq \ell}\left|\varphi\left(y_{j}\right)\right| \geq \mathrm{N}(\varphi) \exp \left\{-c(D \log A)^{\varrho_{0}}\right\}
$$

with

$$
\varrho_{0}=\frac{\ell g}{\ell-2 g(g-1)} .
$$


Proof. To begin with, consider the case $g=1$. We may write $\mathcal{A}=$ $\mathcal{E}$, using the notation of section 3. Then $\varphi(z)=\lambda z$ with $\lambda=\mathrm{N}(\varphi)$. By assumption we have $\ell \geq 1$. For simplicity we write $y$ for $y_{1}$ and $\gamma$ for $\gamma_{1}$. The number $y$ is not a pole of the Weierstraß $\wp$-function, and $\wp(y)$ is an algebraic number of height $\mathrm{h}(\wp(y)) \leq \mathrm{h}(\gamma)$. Hence $|\wp(y)| \leq e^{D \mathrm{~h}(\gamma)} \leq A^{D}$. Since $\wp$ has a double pole at 0 , we deduce

$$
|y| \geq c_{1} A^{-D / 2}
$$

for some positive constant $c_{1}<1$ (which depends only on $\mathcal{E}$, that is of $g_{2}$ and $\left.g_{3}\right)$. This provides the desired result with $c=(1 / 2)-\log c_{1}$ and $\varrho_{0}=1$.

We now assume $g \geq 2$. Apply Theorem 5.2 with $S$ the smallest positive integer which satisfies

$$
S^{\ell-2 g(g-1)} \geq c_{0}^{2 g^{2}}(D \log A)^{g(g-1)} .
$$

Since $S>2$, we deduce

$$
(S / 2)^{\ell-2 g(g-1)}<c_{0}^{2 g^{2}}(D \log A)^{g(g-1)} .
$$

Therefore the parameter $V$ of Theorem 5.2 satisfies the following estimate:

$$
V^{\ell-2 g(g-1)} \leq c_{0}^{2(g+1)(\ell+g)}(D \log A)^{\ell g} .
$$

The choice of $S$ is done in such a way that

$$
c_{0}^{2} V^{g-1} \leq S^{\ell}
$$

Consequently, according to Theorem 5.2 , the set $\Sigma$ has less than $(2 S-1)^{\ell}$ elements. Since $\gamma_{1}, \ldots, \gamma_{\ell}$ are $\mathbb{Z}$-linearly independent, equality $\operatorname{Card} \mathcal{S}=$ Card $\Sigma$ holds, and we deduce that there exists $s^{0} \in \mathbb{Z}^{\ell}$ satisfying

$$
\max _{1 \leq j \leq \ell}\left|s_{j}^{0}\right|<S \quad \text { and } \quad\left|\varphi\left(s_{1}^{0} y_{1}+\cdots+s_{\ell}^{0} y_{\ell}\right)\right|>\mathrm{N}(\varphi) e^{-V}
$$

On the other hand the upper bound

$$
\left|\varphi\left(s_{1}^{0} y_{1}+\cdots+s_{\ell}^{0} y_{\ell}\right)\right| \leq \ell S \max _{1 \leq j \leq \ell}\left|\varphi\left(y_{j}\right)\right|
$$

plainly holds. Since $V+\log (\ell S)<c(D \log A)^{\varrho_{0}}$, the conclusion of Corollary 5.3 follows. 
Corollary 5.4. Let $\mathcal{A}$ be a simple Abelian variety over a number field $K$, of dimension $g$. Let $\gamma_{1}, \ldots, \gamma_{\ell}$ be $\mathbb{Z}$-linearly independent elements of $\mathcal{A}(K)$, with $\ell>2 g(g-1)$. For $1 \leq j \leq \ell$, let $y_{j} \in \mathcal{L}_{\mathcal{A}}(K)$ satisfy $\exp _{\mathcal{A}} y_{j}=$ $\gamma_{j}$. Further let $\omega \in \Omega_{\mathcal{A}}$ be a period of $\exp _{\mathcal{A}}$. There exists a constant $c^{\prime}>0$ such that, for any linear form $\varphi \in \operatorname{Hom}_{\mathbb{C}}\left(T_{\mathcal{A}}(\mathbb{C}), \mathbb{C}\right)$ satisfying $0 \neq \varphi(\omega) \in$ $\mathbb{Z}$, we have

$$
\max _{1 \leq j \leq \ell}\left\|\varphi\left(y_{j}\right)\right\| \geq \mathrm{N}(\varphi) \exp \left(-c^{\prime} N^{2 \varrho_{0}}\right)
$$

with

$$
N=\max \{1, \mathrm{~N}(\varphi)\} \quad \text { and } \quad \varrho_{0}=\frac{\ell g}{\ell-2 g(g-1)}
$$

Proof. Define $s_{0}=\varphi(\omega)$. For $1 \leq j \leq \ell$, let $s_{j} \in \mathbb{Z}$ be an integer at minimal distance of $\varphi\left(y_{j}\right)$, and let

$$
\tilde{y}_{j}=s_{0} y_{j}-s_{j} \omega
$$

so that $\varphi\left(\tilde{y}_{j}\right)=s_{0}\left(\varphi\left(y_{j}\right)-s_{j}\right)$ and $\left|\varphi\left(\tilde{y}_{j}\right)\right|=\left|s_{0}\right| \cdot\left\|\varphi\left(y_{j}\right)\right\|$. The points $\tilde{\gamma}_{j}=\exp _{\mathcal{A}} y_{j},(1 \leq j \leq \ell)$ are linearly independent in $\mathcal{A}(K)$.

Since the final constant $c^{\prime}$ depends on $\omega, y_{1}, \ldots, y_{\ell}$, we may bound $\max _{0 \leq j \leq \ell}\left|s_{j}\right|$ by $c^{\prime \prime} N$, with some suitable constant $c^{\prime \prime}$, and apply Corollary 5.3 with $D=c^{\prime \prime}, \log A=c^{\prime \prime} N^{2}$. Corollary 5.4 easily follows.

We now deduce from Corollary 5.4 a density measure for rational points on an Abelian variety. We need only the condition $\ell>2 g(g-1)$. The estimate is weak compared with Theorem 1.3 , but it is non trivial for $g>1$.

Corollary 5.5. Let $\mathcal{A}$ be a simple Abelian variety of dimension $g$ over a real number field $K \subset \mathbb{R}$. Let $\Gamma$ be a subset of $\mathcal{A}(K)$ of rank $\ell \geq$ $2 g^{2}-2 g+1$. Define

$$
\theta_{0}=\frac{1}{2 \varrho_{0}}=\frac{1}{2 g}-\frac{g-1}{\ell} .
$$

Then there exists a positive constant $C$ such that, for any $h \geq e$ and any $\zeta \in \mathcal{A}(\mathbb{R})^{0}$, the system of inequalities

$$
\operatorname{dist}(\zeta, \gamma) \leq C(\log h)^{-\theta_{0}}, \quad \hat{\mathrm{h}}(\gamma) \leq h
$$

has a solution $\gamma \in \mathcal{A}(K)$. 
Proof. Since the subgroup $\Gamma$ of $\mathcal{A}(K)$ is finitely generated of rank $\ell$, the subgroup $Y=\exp _{\mathcal{A}, \mathbb{R}}^{-1}(\Gamma)$ of $T_{\mathcal{A}}(\mathbb{R})$ is of rank $\ell+g$. Let $\omega_{1}, \ldots, \omega_{g}, y_{1}, \ldots, y_{\ell}$ be a basis of $Y$ over $\mathbb{Z}$, with $\omega_{i} \in \Omega_{\mathcal{A}, \mathbb{R}}$ for $1 \leq i \leq g$. For each nonzero $\varphi \in$ $\Omega_{\mathcal{A}, \mathbb{R}}^{*}$, one at least of the integers $\varphi\left(\omega_{i}\right),(1 \leq i \leq g)$ does not vanish. Therefore we may apply Corollary 5.4. On the lattice $\Omega_{\mathcal{A}, \mathbb{R}}^{*}$ in $\operatorname{Hom}_{\mathbb{R}}\left(T_{\mathcal{A}}(\mathbb{R}), \mathbb{R}\right)$, the two norms

$$
\max \left\{\left|\varphi\left(\omega_{1}\right)\right|, \ldots,\left|\varphi\left(\omega_{g}\right)\right|\right\} \quad \text { and } \mathrm{N}(\varphi)
$$

are equivalent. We deduce that condition (i) of Lemma 2.2 is satisfied with

$$
E=T_{\mathcal{A}}(\mathbb{R}), \quad \Omega=\Omega_{\mathcal{A}, \mathbb{R}}, \quad n=g, \quad m=\ell
$$

and with

$$
F(S)=\exp \left(C_{1} S^{2 \varrho_{0}}\right)
$$

for some positive constant $C_{1}$. The inverse function $G$ of $F$ is bounded from below by

$$
G(T) \geq C_{2}(\log \log T)^{1 / 2 \varrho_{0}}
$$

\section{$\S 6$. Algebraic Diophantine approximation on Abelian varieties}

In this section we prove a non-homogeneous estimate of Diophantine approximation which will enable us to complete the proof of Theorem 1.3.

THEOREM 6.1. Let $\mathcal{A}$ be a simple Abelian variety over a number field $K_{0}$. Let $g$ be the dimension of $\mathcal{A}$, and let $\lambda$ be a positive integer with $\lambda>$ $2 g^{2}$. There exists a constant $c>0$ with the following property. Let $K$ be a finite extension of $K_{0}$, with $[K: \mathbb{Q}]=D$. Let $m$ be a positive integer and $y_{1}, \ldots, y_{m}$ be $\mathbb{Z}$-linearly independent elements in $\mathcal{L}_{\mathcal{A}}(K)$. For $1 \leq j \leq m$ define $\gamma_{j}=\exp _{\mathcal{A}} y_{j}$. Put $Y=\mathbb{Z} y_{1}+\cdots+\mathbb{Z} y_{m}$. Define a real number $A \geq e$ by

$$
\log A=\max _{1 \leq j \leq m} \max \left\{1, \hat{\mathrm{h}}\left(\gamma_{j}\right),(e / D)\left|y_{j}\right|^{2}\right\} .
$$

Let $\beta_{1}, \ldots, \beta_{m}$ be elements in $K$, not all of which are zero. Define a real number $B \geq e$ by

$$
\log B=\max _{1 \leq j \leq m} \max \left\{1, \mathrm{~h}\left(\beta_{j}\right)\right\}
$$

Let $\varphi \in \operatorname{Hom}_{\mathbb{C}}\left(T_{\mathcal{A}}(\mathbb{C}), \mathbb{C}\right)$ be a nonzero linear form such that

$$
\varphi\left(Y \cap \Omega_{\mathcal{A}}\right) \subset \mathbb{Z} \quad \text { and } \operatorname{rank}_{\mathbb{Z}}\left(Y / Y \cap \Omega_{\mathcal{A}} \cap \operatorname{Ker} \varphi\right)=\lambda .
$$


Then

$$
\max _{1 \leq j \leq m}\left|\varphi\left(y_{j}\right)-\beta_{j}\right| \geq \exp \left(-c(D \log A)^{\varrho g}(D \log B)^{\varrho}\right)
$$

with

$$
\varrho=\frac{\lambda}{\lambda-2 g^{2}} .
$$

Proof. We assume that the hypotheses of Theorem 6.1 are satisfied. We denote by $c_{1}$ a sufficiently large number, which depends on the Abelian variety $\mathcal{A}$, on the number field $K_{0}$, and also on $\lambda$, but not on $K, y_{1}, \ldots, y_{m}$, $\varphi$, nor on $\beta_{1}, \ldots, \beta_{m}$. In particular $c_{1}$ does not depend on $D, A$ and $B$. The parameter $m$ is bounded in terms of $\lambda$ and $g$ :

$$
0 \leq m-\lambda \leq 2 g-2
$$

because $Y \cap \Omega_{\mathcal{A}} \cap \operatorname{Ker} \varphi$ is a discrete subgroup of the hyperplane $\operatorname{Ker} \varphi$.

Let $S$ be the smallest positive integer which satisfies

$$
S^{\lambda-2 g^{2}}>\left(c_{1}^{8} D \log A\right)^{g^{2}}(D \log B)^{g} .
$$

Next we define

$$
U_{1}=\left(c_{1}^{4} S^{2} D \log A\right)^{g}(D \log B) \quad \text { and } \quad V_{1}=c_{1} U_{1}
$$

It easily follows that

$$
(S / 2)^{\lambda-2 g^{2}} \leq\left(c_{1}^{8} D \log A\right)^{g^{2}}(D \log B)^{g}
$$

and

$$
U_{1} \leq\left(c_{1}^{8} D \log A\right)^{g \varrho}(D \log B)^{\varrho} .
$$

Assume

$$
\max _{1 \leq j \leq m}\left|\varphi\left(y_{j}\right)-\beta_{j}\right|<e^{-c_{1} V_{1}}
$$

We shall deduce a contradiction, which will complete the proof of Theorem 6.1 with $c=c_{1}^{8 \varrho g+2}$.

We use the notation of Theorem 2.1 of [19] with

$$
\begin{gathered}
G=\mathbb{G}_{\mathrm{a}} \times \mathcal{A}, \quad d_{0}=1, \quad d_{1}=0, \quad d_{2}=g, \quad d=g+1, \quad n=1, \\
W=\mathcal{W}^{\prime}=\{0\}, \quad \ell_{0}=0, \quad r=r_{3}=g, \quad r_{1}=r_{2}=0,
\end{gathered}
$$


while $\mathcal{V}^{\prime}$ is the hyperplane of $\mathbb{C} \times T_{\mathcal{A}}(\mathbb{C})$ of equation $z_{0}=\varphi(z)$. Further we define

$$
\begin{gathered}
B_{1}=B, \quad \log A_{1}=c_{1} S^{2} \log A, \quad E=e, \quad M=S^{m}, \\
T_{0}=\left[U_{1} / D \log B\right] \quad \text { and } \quad T_{1}=\left[U_{1} / D \log A_{1}\right]=\left[U_{1} / c_{1} S^{2} D \log A\right] .
\end{gathered}
$$

One deduces

$$
\left(T_{0}+1\right)\left(T_{1}+1\right)^{g} \geq c_{1} V_{1}^{g} .
$$

Define $\eta_{1}, \ldots, \eta_{m}$ in $K \times \mathcal{L}_{\mathcal{A}}(K) \subset \mathbb{C} \times T_{\mathcal{A}}(\mathbb{C})$ and $\eta_{1}^{\prime}, \ldots, \eta_{m}^{\prime}$ in $\mathcal{V}^{\prime} \subset$ $\mathbb{C} \times T_{\mathcal{A}}(\mathbb{C})$ as follows

$$
\eta_{j}=\left(\beta_{j}, y_{j}\right) \quad \text { and } \quad \eta_{j}^{\prime}=\left(\varphi\left(y_{j}\right), y_{j}\right), \quad(1 \leq j \leq m) .
$$

Next define $2 M$ elements of $\mathbb{C} \times T_{\mathcal{A}}(\mathbb{C})$, which are indexed by $s=$ $\left(s_{1}, \ldots, s_{m}\right) \in \mathbb{Z}^{m}$ with $0 \leq s_{j}<S,(1 \leq j \leq m)$ :

$$
\eta_{s}=s_{1} \eta_{1}+\cdots+s_{m} \eta_{m}, \quad \eta_{s}^{\prime}=s_{1} \eta_{1}^{\prime}+\cdots+s_{m} \eta_{m}^{\prime} .
$$

Hence

$$
\max _{s}\left|\eta_{s}-\eta_{s}^{\prime}\right| \leq e^{-V_{1}}
$$

We consider the following subset of $K \times \mathcal{A}(K)$ :

$$
\Sigma=\left\{\left(s_{1} \beta_{1}+\cdots+s_{m} \beta_{m}, s_{1} \gamma_{1}+\cdots+s_{m} \gamma_{m}\right) ; 0 \leq s_{j}<S,(1 \leq j \leq m)\right\},
$$

and its two projections

$$
\Sigma_{0}=\left\{s_{1} \beta_{1}+\cdots+s_{m} \beta_{m} ; 0 \leq s_{j}<S,(1 \leq j \leq m)\right\} \subset K
$$

and

$$
\Sigma_{1}=\left\{s_{1} \gamma_{1}+\cdots+s_{m} \gamma_{m} ; 0 \leq s_{j}<S,(1 \leq j \leq m)\right\} \subset \mathcal{A}(K) .
$$

The assumption $\varphi\left(Y \cap \Omega_{\mathcal{A}}\right) \subset \mathbb{Z}$ will be used as follows:

$$
\text { If } s=\left(s_{1}, \ldots, s_{m}\right) \in \mathbb{Z}^{m} \text { satisfies the three properties }
$$

$$
\max _{1 \leq j \leq m}\left|s_{j}\right|<S, \quad s_{1} \beta_{1}+\cdots+s_{m} \beta_{m}=0 \quad \text { and } \quad s_{1} \gamma_{1}+\cdots+s_{m} \gamma_{m}=0,
$$

then

$$
\left|\varphi\left(s_{1} y_{1}+\cdots+s_{m} y_{m}\right)\right| \leq m S \max _{s}\left|\eta_{s}-\eta_{s}^{\prime}\right| \leq m S e^{-V_{1}}<1
$$


hence

$$
s_{1} y_{1}+\cdots+s_{m} y_{m} \in Y \cap \Omega_{\mathcal{A}} \cap \operatorname{Ker} \varphi
$$

Given the definition of $\lambda$, the number of such $s \in \mathbb{Z}^{m}$ is at most $(2 S)^{m-\lambda}$.

The algebraic subgroups $G^{*}$ of $G=\mathbb{G}_{\mathrm{a}} \times \mathcal{A}$, distinct from $G$, are $\{0\}$, $\{0\} \times \mathcal{A}$ and $\mathbb{G}_{\mathrm{a}} \times\{0\}$. We give accordingly three lower bounds for the cardinality of the projection of $\Sigma$ on $G(K) / G^{*}(K)$.

a) The case $G^{*}=\{0\}$.

We claim

$$
\text { Card } \Sigma \geq 2^{-g} c_{1} T_{0} T_{1}^{g}
$$

Indeed, from the choice of parameters and conditions (6.2) and (6.3) we deduce

$$
S^{\lambda} \geq 2^{g} c_{1} T_{0} T_{1}^{g}
$$

Using the assumption $\varphi\left(Y \cap \Omega_{\mathcal{A}}\right) \subset \mathbb{Z}$ as shown above, we deduce

$$
\operatorname{Card} \Sigma \geq S^{m} /(2 S)^{m-\lambda} \geq 2^{-g} c_{1} T_{0} T_{1}^{g}
$$

b) The case $G^{*}=\{0\} \times \mathcal{A}$.

We want to check

$$
\operatorname{Card} \Sigma_{0} \geq c_{1} T_{0}
$$

The set

$$
\mathcal{S}_{1}=\left\{s=\left(s_{1}, \ldots, s_{m}\right) \in \mathbb{Z}^{m} ; \max _{1 \leq j \leq m}\left|s_{j}\right|<S, s_{1} \beta_{1}+\cdots+s_{m} \beta_{m}=0\right\}
$$

satisfies

$$
\left(\operatorname{Card} \mathcal{S}_{1}\right)\left(\operatorname{Card} \Sigma_{0}\right) \geq S^{m} .
$$

For $s \in \mathcal{S}_{1}$, we have $\left|\varphi\left(s_{1} y_{1}+\cdots+s_{m} y_{m}\right)\right| \leq m S e^{-V_{1}}$. Since $\left(\beta_{1}, \ldots, \beta_{m}\right) \neq$ $(0, \ldots, 0)$, we deduce from Liouville's inequality

$$
B^{-D} \leq \max _{1 \leq j \leq m}\left|\beta_{j}\right| \leq \max _{1 \leq j \leq m}\left|\varphi\left(y_{j}\right)-\beta_{j}\right|+\mathrm{N}(\varphi)((1 / e) D \log A)^{1 / 2}
$$

Therefore the parameter $V$ of Theorem 5.2 satisfies

$$
m S e^{-V_{1}} \leq \mathrm{N}(\varphi) e^{-V}
$$

and we deduce from that theorem

$$
\operatorname{Card}\left\{s_{1} \gamma_{1}+\cdots+s_{m} \gamma_{m} ; s \in \mathcal{S}_{1}\right\} \leq c_{1}^{g+1}\left(c_{1}^{2} S^{2} D \log A\right)^{g(g-1)} .
$$


From the assumption $\varphi\left(Y \cap \Omega_{\mathcal{A}}\right) \subset \mathbb{Z}$ we deduce

$$
\operatorname{Card} \mathcal{S}_{1} \leq(4 S)^{m-\lambda} c_{1}^{g+1}\left(c_{1}^{2} S^{2} D \log A\right)^{g(g-1)},
$$

which yields

$$
\operatorname{Card} \Sigma_{0} \geq c_{1}^{-g-2} S^{\lambda-2 g^{2}+2 g}\left(c_{1}^{2} D \log A\right)^{-g(g-1)} .
$$

Our claimed lower bound for Card $\Sigma_{0}$ follows from the estimate

$$
S^{\lambda-2 g^{2}+2 g}>c_{1}^{g+3} T_{0}\left(c_{1}^{2} D \log A\right)^{g(g-1)} .
$$

c) The case $G^{*}=\mathbb{G}_{\mathrm{a}} \times\{0\}$.

Now we are going to check

$$
\operatorname{Card} \Sigma_{1} \geq c_{1} T_{1}^{g}
$$

Put $\Gamma=\exp _{\mathcal{A}}(Y)$. If $\ell$ is the rank of $\Gamma$, then $\operatorname{Card} \Sigma_{1} \geq S^{\ell}$. Now from the definition of $\lambda$ follows

$$
\lambda-\ell=\operatorname{rank}_{\mathbb{Z}}\left(Y \cap \Omega_{\mathcal{A}} / Y \cap \Omega_{\mathcal{A}} \cap \operatorname{Ker} \varphi\right) \leq \operatorname{rank}_{\mathbb{Z}} \Omega_{\mathcal{A}}=2 g .
$$

Since (6.3) implies

$$
U_{1}>c_{1}(D \log B) S^{2 g}
$$

we deduce $T_{0} \geq S^{2 g}$ and (using (6.4))

$$
\operatorname{Card} \Sigma_{1} \geq S^{\ell} \geq S^{\lambda-2 g} \geq c_{1} T_{0} T_{1}^{g} S^{-2 g} \geq c_{1} T_{1}^{g} .
$$

These estimates show that the conclusion of Theorem 2.1 of [19] does not hold. This completes the proof of Theorem 6.1.

We now deduce from Theorem 6.1 the following corollary, which obviously implies Theorem 1.3 (just take $\Gamma=\mathcal{A}(K)$ ):

Corollary 6.5. Let $\mathcal{A}$ be a simple Abelian variety of dimension $g$ over a real number field $K \subset \mathbb{R}$. Let $\Gamma$ be a subset of $\mathcal{A}(K)$ of rank $\ell \geq 2 g^{2}$. Then there exist two positive constants $C_{1}$ and $C_{2}$ such that, for any $h \geq e$ and any $\zeta \in \mathcal{A}(\mathbb{R})^{0}$, there exists $\gamma \in \mathcal{A}(K)$ of height $\hat{\mathrm{h}}(\gamma) \leq h$ satisfying

$$
\operatorname{dist}(\zeta, \gamma) \leq C_{1} \exp \left\{-C_{2}(\log h)^{\theta}\right\} \quad \text { with } \theta=1-\frac{2 g^{2}}{\ell+1}
$$


Proof. When $\ell$ is the rank of $\Gamma$, the subgroup $\exp _{\mathcal{A}, \mathbb{R}}^{-1}(\Gamma)$ of $T_{\mathcal{A}}(\mathbb{R})$ is of rank $m=\ell+g$. Let $\omega_{1}, \ldots, \omega_{g}$ be a basis of $\Omega_{\mathcal{A}, \mathbb{R}}$ and let $y_{1}, \ldots, y_{\ell}$ be elements in $\exp _{\mathcal{A}, \mathbb{R}}^{-1}(\Gamma)$ such that $\omega_{1}, \ldots, \omega_{g}, y_{1}, \ldots, y_{\ell}$ are $\mathbb{Z}$-linearly independent. Define a subgroup $Y$ of finite index in $\exp _{\mathcal{A}, \mathbb{R}}^{-1}(\Gamma)$ by

$$
Y=\mathbb{Z} \omega_{1}+\cdots+\mathbb{Z} \omega_{g}+\mathbb{Z} y_{1}+\cdots+\mathbb{Z} y_{\ell}
$$

Let $\varphi \in \operatorname{Hom}_{\mathbb{R}}\left(T_{\mathcal{A}}(\mathbb{R}), \mathbb{R}\right)$ be a nonzero linear form on $T_{\mathcal{A}}(\mathbb{R})$ such that $\varphi\left(\Omega_{\mathcal{A}, \mathbb{R}}\right) \subset \mathbb{Z}$. Define rational integers $\beta_{1}, \ldots, \beta_{m}$ as follows: for $1 \leq i \leq g$, let $\beta_{i}=\varphi\left(\omega_{i}\right)$; for $g<j \leq m$, we choose for $\beta_{j}$ a rational integer at minimal distance of $\varphi\left(y_{j-g}\right)$. From our assumption $\varphi \neq 0$, we deduce that one at least of the numbers $\beta_{1}, \ldots, \beta_{g}$ does not vanish. Since

$$
\operatorname{rank}_{\mathbb{Z}}\left(Y \cap \Omega_{\mathcal{A}} \cap \operatorname{Ker} \varphi\right)=g-1
$$

we may apply the estimate of Theorem 6.1 with $\lambda=\ell+1$. We deduce that there exist positive constants $S_{0}$ and $C^{\prime}$ such that, for any nonzero $\varphi \in \Omega_{\mathcal{A}, \mathbb{R}}^{*}$ for which the number $S=\max \left\{\left|\varphi\left(\omega_{1}\right)\right|, \ldots,\left|\varphi\left(\omega_{g}\right)\right|\right\}$ satisfies $S \geq S_{0}$, the inequality

$$
\max _{1 \leq j \leq m}\left\|\varphi\left(y_{j}\right)\right\| \geq 1 / F(S)
$$

holds with

$$
F(S)=\exp \left\{C^{\prime}(\log S)^{\varrho}\right\} \quad \text { and } \quad \varrho=\frac{\ell+1}{\ell+1-2 g^{2}},
$$

Applying Lemma 2.2 to the $\mathbb{R}$-vector space $T_{\mathcal{A}}(\mathbb{R})$ yields the conclusion with $\theta=1 / \varrho$.

\section{REFERENCES}

[1] D. Bertrand et D.W. Masser, Linear forms in elliptic integrals, Invent. Math., 58 (1980), no. 3, 283-288.

[2] J.-L. Colliot-Thélène, A. N. Skorobogatov and P. Swinnerton-Dyer, Double fibres and double covers: paucity of rational points, Acta Arith., 79 (1997), no. 2, 113-135.

[3] N. Hirata-Kohno, Approximations simultanées sur les groupes algébriques commutatifs, Compositio Math., 86 (1993), no. 1, 69-96.

[4] J. Huisman, Heights on Abelian varieties, in Diophantine Approximation and Abelian varieties (Soesterberg, 1992) (B. Edixhoven and J.-H. Evertse, eds.), Chap V, Lecture Notes in Math., 1566, Springer-Verlag (1993), pp. 51-61. 
[5] S. Lang, Elliptic curves: Diophantine analysis, Grundlehren der Mathematischen Wissenschaften [Fundamental Principles of Mathematical Sciences], 231, Springer-Verlag, Berlin-New York, 1978.

[6] _ Fundamental of Diophantine geometry, Springer-Verlag, New York-Berlin, 1983.

[7] , Number theory III Diophantine geometry, Encyclopaedia of Mathematical Sciences, 60, Springer-Verlag, Berlin, 1991. Corrected second printing: Survey of Diophantine Geometry, 1997.

[8] D. W. Masser, Elliptic functions and transcendence, Lecture Notes in Mathematics, 437, Springer-Verlag, Berlin-New York, 1975.

[9] B. Mazur, The topology of rational points, Experiment. Math., 1 (1992), no. 1, 35-45.

[10] D. Roy, Simultaneous approximation in number fields, Invent. Math., 109 (1992), no. $3,547-556$.

[11] Th. Schneider, Einführung in die transzendenten Zahlen, Springer-Verlag, BerlinGöttingen-Heidelberg, 1957. Introduction aux nombres transcendants; Traduit de l'allemand par P. Eymard. Gauthier-Villars, Paris 1959.

[12] J-P. Serre, Lectures on the Mordell-Weil theorem, Translated from the French and edited by Martin Brown from notes by Michel Waldschmidt, Aspects of Mathematics, E15, Friedr. Vieweg \& Sohn, Braunschweig, 1989. Second Ed., 1990.

[13] J. Silverman, The arithmetic of elliptic curves, Graduate Texts in Mathematics, 106, Springer-Verlag, New York-Berlin, 1986. Advanced topics in the Arithmetic of Elliptic Curves, Graduate Texts in Mathematics, 151, Springer-Verlag, New York, 1994.

[14] M. Waldschmidt, Nombres transcendants et groupes algébriques, With appendices by Daniel Bertrand and Jean-Pierre Serre, Astérisque, 69-70, Société Mathématique de France, Paris, 1979, 218 pp. Second edition 1987.

[15] — Dependence of logarithms of algebraic points, in Number theory, Vol. II (Budapest, 1987), Colloq. Math. Soc. János Bolyai, 51, North-Holland, Amsterdam (1990), pp. 1013-1035.

[16] On the transcendence methods of Gel'fond and Schneider in several variables, in New advances in transcendence theory (Durham, 1986), Cambridge Univ. Press, Cambridge-New York (1988), pp. 375-398.

[17] Densité de points rationnels sur un groupe algébrique, Experiment. Math., 3 (1994), no. 4, 329-352 et 4 (1995), no. 3, 255.

[18] - Topologie des points rationnels, Cours de Troisième Cycle 1994/95, Univ. P. et M. Curie, 168 pp. http://www.math.jussieu.fr/ $/$ miw/TPR.html.

[19] - Approximation diophantienne dans les groupes algébriques commutatifs (I): Une version effective du théorème du sous-groupe algébrique, J. reine angew. Math., 493 (1997), 61-113.

[20] — Approximation simultanée par des produits de puissances de nombres algébriques, Acta Arith., 79 (1997), no. 2, 137-162. 
Université P. et M. Curie (Paris VI)

Institut Mathématique de Jussieu

Problèmes Diophantiens, Case 247

4, Place Jussieu

$F$ - 75252 PARIS CEDEX 05

miw@math.jussieu.fr 